\section{CUIDADOS GENERALES A PACIENTES CON VENTILACIÓN MECÁNICA NO INVASIVA}

GENERAL CARE PATIENTS WITH NON-INVASIVE MECHANICAL VENTILATION

Fenoll Jimenez, José Joaquín(a)

García Navalón, Antonio Francisco(b)

Marchán Beneyto, Alfredo(c)

(a) Enfermero del Hospital Marina Baixa Villajoyosa. Alicante.

(b) Enfermero del Hospital General de Albacete.

(c) Enfermero de Atención Primaria Departamento Marina Baixa. Villajoyosa.

Contacto: jjfenol@hotmail.com 


\section{RESUMEN}

Titulo: Cuidados generales a pacientes con ventilación mecánica no invasiva.

\section{Objetivos:}

Homogeneizar los criterios de actuación de Enfermería para mejorar la calidad asistencial.

Proporcionar una herramienta de trabajo para los trabajadores de nueva incorporación fomentando el trabajo en equipo y mejorando la organización y planificación de los cuidados hacia el paciente.

\section{Método:}

Estudio descriptivo elaborado tras una revisión bibliográfica como apoyo a la experiencia profesional.

\section{Resultados:}

La descripción del procedimiento que se emplea, nos sirve de guía para el buen funcionamiento del proceso.

\section{Conclusiones:}

La implicación de la enfermería es de suma importancia en el proceso del paciente que precisa de Ventilación Mecánica no Invasiva (VMNI).

La comparación de los artículos con el resultado obtenido y la puesta en común, nos ha llevado a la conclusión de la importancia de establecer unas bases que sirvan de motivación a los profesionales sanitarios para conseguir el mayor éxito posible en los objetivos planteados.

Una adecuada secuencia de técnicas aplicadas correctamente, condicionan el éxito de la VMNI.

Es imprescindible que enfermería tenga los conocimientos y las habilidades necesarios para un correcto inicio de la VMNI.

\section{Palabras claves:}

Enfermería, cuidados, Ventilación Mecánica no Invasiva (VMNI), oxigenación, ventilación. 


\section{ABSTRACT}

Title: General care patients with noninvasive mechanical ventilation.

\section{Objectives:}

Mix the performance criteria to improve nursing care quality. Provide a working tool for fostering new workers teamwork and improving the organization and planning of care to the patient.

\section{Method:}

A descriptive study was developed following a literature review to support the experience.

\section{Results:}

The description of the procedure used, serves as a guide for the proper functioning of the process.

\section{Conclusions:}

The involvement of nursing is of utmost importance in the process of patients who need Noninvasive Mechanical Ventilation (NIV).

The comparison of items with the result and sharing has led us to conclude the importance of establishing a foundation that will serve as motivation to healthcare professionals to achieve the greatest possible success in the objectives.

A proper sequence of techniques properly applied, determine the success of NIV.

It is imperative that nurses have the knowledge and skills necessary for proper initiation of NIV.

\section{Keywords:}

Nursing, Care, Noninvasive mechanical ventilation (NIV), oxygenation, ventilation. 


\section{INTRODUCCIÓN}

Desde hace más de treinta años se utiliza la ventilación mecánica (VM) como soporte respiratorio en pacientes con insuficiencia respiratoria aguda (IRA). Este soporte se aplicaba a través de una vía aérea artificial, es decir, ventilación mecánica invasiva (VMI). El modo ventilatorio VMNI inicial con modo CPAP fue introducido desde 1912 por Buñuel. La ventilación mecánica no invasiva (VMNI) comenzó a utilizarse en la década de los 70, reportando desde entonces importantes ventajas para el paciente ${ }^{(1)}$. En los últimos años hemos visto incrementado el uso de la VMNI sobre todo en unidades de cuidados intensivos. A pesar de ello, todavía hoy este tipo de asistencia respiratoria sigue siendo una gran desconocida por parte de los profesionales, poco familiarizados con la técnica. Por este motivo queremos, por medio de este trabajo, basado en la revisión bibliográfica acercar a los profesionales de enfermería a la VMNI, tan beneficiosa para el paciente respiratorio.

\section{DESCRIPCIÓN DEL PROCEDIMIENTO}

Se podría definir la Ventilación No Invasiva (VNI) como el conjunto de técnicas empleadas para aumentar la ventilación alveolar sin requerir la creación de una vía artificial (IOT, traqueotomía)

\section{Fundamentos Fisiológicos de la VMNI-CPAP:}

Su utilización se basa en que el cierre progresivo de los alveolos, y el shunt alveolocapilar ocurren esencialmente al final de la espiración. Al aplicar una presión positiva mantenida al finalizar la espiración (PEEP) permite disminuir el colapso alveolar, facilitar la apertura parcialmente de los alveolos colapsados y mejorar el intercambio gaseoso relajando la musculatura respiratoria(2)

Se pueden aplicar diferentes modos ventilatorios, siendo la ventilación a presión positiva la utilizada con mayor frecuencia CPAP (Continuos Positive Airea Presura). Desde el personal de enfermería la observación a pie de cama es fundamental para el éxito de la técnica. En las primeras horas supone un aumento de las cargas de trabajo y requiere que el profesional esté 
familiarizado con el proceso. Muchas son las ventajas y escasas y leves las complicaciones que pueden producirse, por lo que la VMNI gana peso en la asistencia respiratoria en los hospitales.

Tiene un amplio abanico de indicaciones, entre las que destacan la IRA y reagudización del EPOC $(3,4)$

\section{Indicaciones:}

- Insuficiencia respiratoria hipercápnica de origen pulmonar o extrapulmonar.

- EPOC (enfermedad pulmonar obstructiva crónica)

- SAOS (síndrome apnea obstructiva del sueño)

- Enfermedades neuromusculares.

- Insuficiencia respiratoria hipoxémica.

- SDRA (síndrome distrés respiratorio agudo)

- Neumonía grave.

- Edema agudo de pulmón.

- Pacientes con fracaso postextubación.

- Método de weaning (destete)

- Otros: gastroscopias...

\section{Ventajas:}

- Intercambio de gases: oxigenación-ventilación.

- Control de síntomas: disnea, alteraciones del sueño.

- Musculatura respiratoria: mejora la fatiga, favoreciendo el reposo de los músculos respiratorios.

- Mejora la calidad de vida.

- Permite la aplicación de forma intermitente.

- Fácil de retirar y fácil de reinstaurar en caso de que se precise.

- Permite al paciente toser, hablar, expectorar o vomitar.

- Evita el dolor o la molestia derivada del tubo orotraqueal, así como la ansiedad.

- Reduce la necesidad de sedación profunda. 
- Evita la colocación de sondas nasogástricas de forma obligatoria.

- Evita complicaciones del tubo endotraqueal: traumas, intubaciones selectivas y daños en hipofaringe, laringe y tráquea.

- Reduce el tiempo de estancia hospitalaria.

- Evita infecciones nosocomiales y complicaciones postextubación: disfonía, estridor, estenosis traqueales, etc.

- Puede reducir la atrofia de la musculatura respiratoria inducida por la ventilación mecánica.

- Se puede instaurar en estadios relativamente precoces de la insuficiencia respiratoria, al contrario que la intubación orotraqueal.

\section{Inconvenientes: ${ }^{(5)}$}

- Precisa mayor tiempo invertido por el personal de Enfermería en las primeras horas de la instauración de la VMNI.

- Puede provocar complicaciones como distensión gástrica, irritación conjuntival, sequedad de mucosas.

- Puede provocar lesiones de piel sobre todo en nariz, llegando a producir incluso úlceras.

- Intolerancia a la mascarilla.

- Fugas aéreas por mal sellado de la mascarilla.

- Dificultad para humidificar el sistema.

- Dificultad para aspirar secreciones en caso de que sea necesario.

- Es necesario un grado mínimo de colaboración por parte del paciente.

\section{Contraindicaciones: $(6,7)$}

- Intolerancia a la mascarilla.

- Nivel de conciencia disminuido.

- Traumatismo facial.

- Patrón respiratorio inestable.

- Neumotórax.

- Cardiopatía isquémica aguda.

- Hipertensión intracraneal.

- HDA, hipotensión, arritmias ventriculares ... 


\section{TIPOS DE INTERFASES:}

- Mascarilla nasal: tiene como principal particularidad para su uso, que el paciente debe tener la boca cerrada para evitar fugas y conseguir así la ventilación deseada. Esta mascarilla es menos claustrofóbica y se utiliza principalmente en patologías respiratorias crónicas (apnea del sueño, hipercapnia, etc.).

MASCARILLAS NASALES
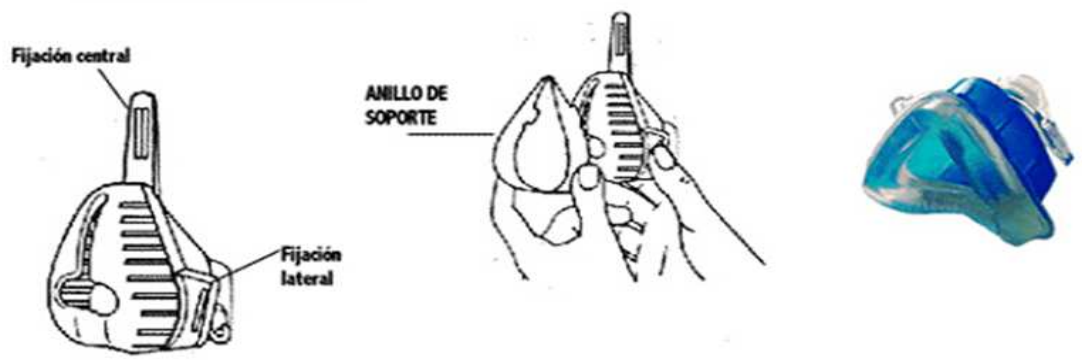

Figura 1. Mascarilla nasal.

- Mascarilla oronasal: se utiliza principalmente en patologías respiratorias agudas (hipoxia, hipercapnia, etc.)

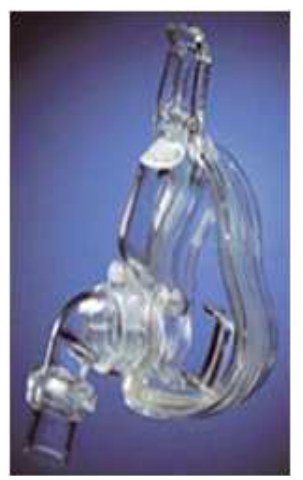

Figura 2. Mascarilla oronasal

- Mascarilla facial completa: utiliza un sistema de sellado que sella alrededor del perímetro facial, evitando la presión directa sobre las estructuras nasales.

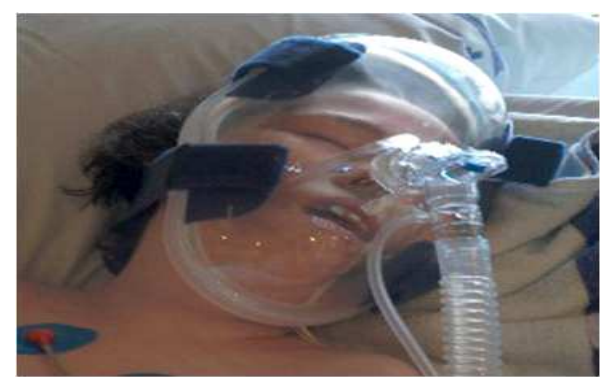

Figura 3. Mascarilla facial completa. 
- Helment (Casco en Inglés): es utilizado para reducir las complicaciones directamente relacionadas con los otros tipos de interfases nombrados anteriormente.

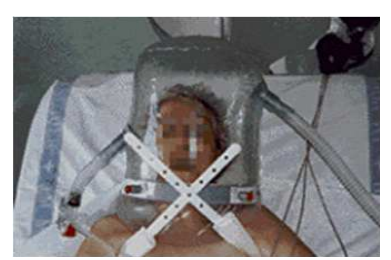

Figura 4. Helment.

\section{TIPOS VENTILATORIOS:}

Actualmente utilizamos 3 tipos de ventiladores, que se denominan:

- $\quad$ Bipap $R$ S/T-D

- Vision $\mathbb{R}$ y

- Quantum $\mathbb{R}$

1.- El sistema Bipap S/T-D \& está diseñado para funcionar en cuatro modalidades diferentes:

- Modo Spontaneous (S): la unidad cicla entre IPAP (inicia un ciclo de Presión Positiva de las vías respiratorias, en respuesta a un esfuerzo inspiratorio espontáneo) y EPAP (se inicia un ciclo de Presión Positiva en la espiración, en respuesta a un esfuerzo espiratorio espontáneo), siguiendo el ritmo respiratorio del paciente.

- Modo Spontaneous/Timed (S/T): la unidad cicla como en el modo (S) pero si el paciente es incapaz de iniciar una respiración la unidad ciclará a IPAP en base a las respiraciones por minuto (mando de control BPM).

- Modo Timed (T): la unidad cicla entre los niveles de IPAP e EPAP en base a la frecuencia respiratoria programada con el mando BPM y el \% de IPAP Time seleccionado.

- Modo CPAP: la unidad mantiene de forma constante un nivel de presión $($ CPAP) $=$ (IPAP).

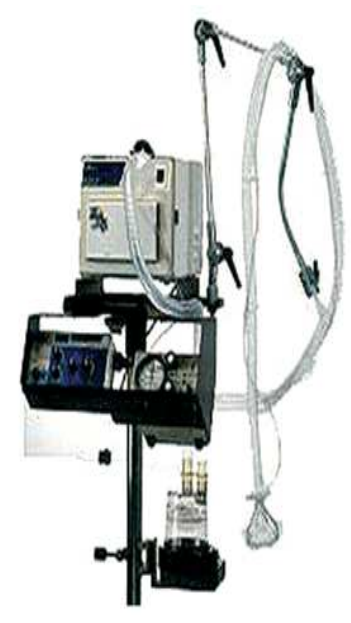


2.- El modelo Vision $\circledast$ puede funcionar en tres modalidades:

- Modo S/T: igual que en el modelo anterior.

- Modo CPAP: igual que en el modelo anterior.

- Modo PAV/T (Proportional Assist Ventilation): es una nueva modalidad que poseen los últimos modelos de Vision, por ser tan novedosa su uso no está muy extendido. El ventilador genera una presión

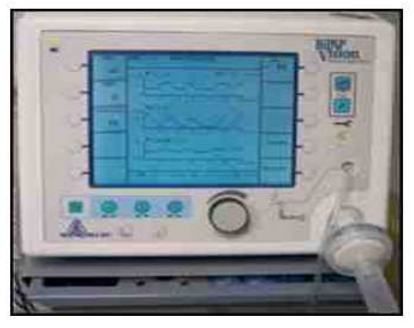
proporcional al esfuerzo del paciente, el esfuerzo del paciente y el grado de asistencia, que programa el médico, es el que determina el volumen que realizará el paciente. La función Time $(T)$, que también posee ésta modalidad, hace que en caso de que el paciente no realice el mínimo de respiraciones programadas el ventilador lo haga en base a dicho ritmo.

\section{3.- Quantum $®$ :}

- Modo spont: igual que en el modelo anterior (S).

- Spont-timed: igual que en el modelo anterior (S/T).

- Modo CPAP: igual que en el modelo anterior (IPAP).

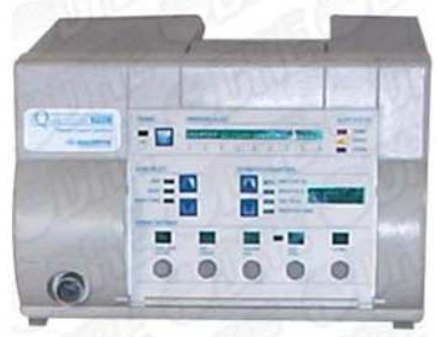

\section{EVIDENCIA DISPONIBLE EN CUIDADOS DE ENFERMERÍA EN PACIENTES CON VENTILACIÓN MECÁNICA NO INVASIVA:}

Los cuidados de enfermería tendrán como finalidad prevenir, disminuir, o resolver en la medida de lo posible las complicaciones e incidencias durante la VMNI $(8,9,10,11)$

\section{A. Antes de iniciar la técnica de VMNI}

- Informar al paciente del procedimiento a realizar, tranquilizarlo y darle confianza.

- Preparar el material y realizar el chequeo del ventilador. Elegir el tipo de interfase más adecuado para el enfermo según la situación clínica de este y las ventajas e inconvenientes de cada una. 
- El médico ajustará los parámetros ventilatorios según la situación del paciente.

- Colocar al paciente en posición semisentado (con la parte superior del cuerpo incorporado por encima de $45^{\circ}$ ), para facilitarle el trabajo respiratorio, disminuir el riesgo de aspiración y conseguir mayor volumen corriente.

- Proteger el arco de la nariz con un apósito hidrocoloidal o hidrocelulares, para evitar que las presiones de la interfase provoquen úlceras por decúbito.

- Hidratar las mucosas y colocar vaselina o pomadas hidratantes hidrosolubles en los labios, la nariz y la mucosa nasal, especialmente, si se utilizara la mascarilla facial, para mitigar la sequedad.

- Colocar la interfase entre dos personas que se colocarán una a cada lado del enfermo. Primero se coloca el arnés por la parte posterior de la cabeza y, con el respirador fuuncionando, se posiciona la mascarilla en su lugar correspondiente, según sea nasal o facial, y después se van ajustando las correas del arnés a la cabeza del paciente hasta que queda la mascarilla bien ajustada consiguiendo la mínima fuga posible.

- Aplicar suavemente la interfase sobre la cara hasta que el paciente se encuentre cómodo y sincronizado con el ventilador. En pacientes muy angustiados podemos dejar que el mismo se aplique la interfase, hasta que pierda el temor.

- Preguntar frecuentemente al enfermo por sus necesidades (posición de la interfase, presencia de dolor, incomodidad, fugas molestas, deseo de expectorar), o posibles complicaciones (aumento de disnea,distensión abdominal, naúseas, vómitos)

\section{B. Actuaciones de Enfermería durante la VMNI:}

- Monitorizar las constantes vitales: presión arterial, frecuencia respiratoria, saturación de $\mathrm{O}_{2}$, ECG, etc. 
- Controlar signos y síntomas de dificultad respiratoria (cianosis distal, disminución de nivel de conciencia, valores anormales de constantes vitales). Sobre todo hay que estar muy atentos en los momentos de conexión y desconexión del sistema de ventilación, ya sean voluntarios (alimentación, hidratación, eliminación de secreciones, etc.) o involuntarios.

- Valorar signos de distensión abdominal y avisar al médico en caso de que dicha distensión dificulte el trabajo respiratorio al elevar el diafragma y comprimir las bases pulmonares, para considerar la posibilidad de colocar sonda nasogástrica.

- Valorar la presencia de molestias pectorales y de intolerancia a la técnica; avisar al médico si se producen.

- Controlar y comunicar en caso necesario, los cambios del estado mental del enfermo (inquietud, confusión, agitación, etc.) que dificultan la consecución de la técnica.

- Anotar la hora y los cambios en los parámetros del ventilador, para valorar su influencia en la evolución del paciente.

- Aportar alimentación e hidratación. Desconectar temporalmente la mascarilla y proporcionar periódicamente el aporte de nutrientes y líquidos, siempre que la situación del enfermo lo permita. La interrupción de la ventilación debe ser lo más breve posible, volviendo a colocar el arnés y la mascarilla al término de la actividad. Agrupar los cuidados para evitar desconexiones innecesarias.

- Enseñar y facilitar la eliminación de secreciones. La hermeticidad de las mascarillas faciales, junto con elevados flujos de $\mathrm{O} 2$, favorecen el acúmulo de secreciones (atelectasias, neumonías, etc...) por lo que es necesario insistir en su movilización y expulsión mediante fisioterapia respiratoria, aspiración de secreciones o facilitando su eliminación activa por parte del enfermo.

- Evitar las úlceras por presión: vigilando las zonas de mayor fricción y presión por el arnés o por la mascarilla, cambiando, si se puede, las 
zonas de sujeción. Controlar el estado de los apósitos de protección y reemplazarlos en caso necesario.

- Hidratar las mucosas: desconectando temporalmente la mascarilla y apagando la alarma sonora de desconexión, aplicar vaselina o pomadas hidratantes hidrosolubles en labios y mucosa mediante torunda de gasas y facilitar el uso de enjuagues bucales, intentando que la desconexión, en caso de utilizar mascarilla facial, sea lo más breve posible. Lubricar los ojos con lágrimas artificiales para prevenir conjuntivitis, úlceras oculares o una incómoda sequedad ocular, debido a las fugas del flujo aéreo.

- Vigilar y controlar las alarmas que aparezcan en el ventilador y corregir su causa.

\section{Actuaciones enfermeras después de la VMNI:}

- Colocar al paciente en posición cómoda: eliminar secreciones si las hubiese, hidratar las mucosas y administrar oxigenoterapia según prescripción y estado respiratorio del enfermo (gafas nasales, mascarilla de Venturi, etc.).

- Recoger el material empleado y proceder a su limpieza y mantenimiento.

\section{COMPLICACIONES POTENCIALES $(11,12,13,14,15)$ :}

$>$ Lesiones dérmicas faciales y necrosis en el puente nasal: debidas a la presión continua que ejercen la mascarilla y el arnés utilizado para mitigar las fugas y mantener las presiones terapéuticas, dando lugar a una hipoxia tisular de las zonas presionadas y a la aparición de úlceras por presión.

$>$ Distensión gástrica: provocada por la acumulación de aire en el estómago causada por la semihermeticidad del circuito, que introduce aire del ventilador sobre todo en cavidad pulmonar, pero también en cámara gástrica. 
$>$ Vómito y aspiración: debido a la hermeticidad que debemos conseguir en el circuito para alcanzar las presiones terapéuticas taponando las salidas anatómicas de nariz, boca o ambas con las mascarillas, la expulsión de los vómitos se ve dificultada, por lo que aumenta el riesgo de aspiración bronquial de éste. Además, el riesgo de vómito se aumenta por la distensión gástrica.

$>$ Conjuntivitis: provocada por las fugas del flujo de aire continuo que se necesita para alcanzar las presiones y que resecan la mucosa ocular facilitando la inflamación e irritación de ésta.

$>$ Intolerancia a la interfase: el paciente puede manifestar cuadros de agitación, inquietud y nerviosismo causados por la sensación de claustrofobia, producida por la colocación de este tipo de mascarilla y arnés en la cabeza, aumentados por el estado de ansiedad que provoca la dificultad respiratoria percibida por el paciente.

$>$ Atelectasias y neumonía: las secreciones se acumulan por la dificultad de expulsión y la sequedad que produce el flujo aéreo del ventilador, que favorece, además, su retención en los alveolos y bronquíolos ${ }^{(16)}$

$>$ Sequedad de mucosa buconasal: el flujo continuo de aire necesario para conseguir las presiones terapéuticas reseca las mucosas, pudiendo provocar irritación y dolor, además de un mayor riesgo de infecciones de flora bacteriana oportunista.

\section{CONCLUSIONES:}

Es absolutamente necesario el entrenamiento del enfermero/a en el manejo de pacientes con VMNI, para lograr el éxito en el tratamiento.

Los pacientes con este tipo de soporte ventilatorio requieren un alto nivel de cuidados "a pié de cama".

Es fundamental la colaboración de los pacientes, por ello la enfermería debe incidir en actuaciones tendentes a disminuir el nivel de ansiedad del paciente y facilitar, en lo posible, su confort. 


\section{BIBLIOGRAFÍA.}

1. Salvador Díaz Lobato, Sagrario Mayoralas Alises. La ventilación mecánica no invasiva moderna cumple 25 años. Original Research Article. Archivos de Bronconeumología, Volume 49, Issue 11, November 2013, Pages 475-479.

2. Antonio Esquinas Rodríguez. Guía esencial de metodología en ventilación mecánica no invasiva / Essential Guide of methodology in non-invasive mechanical ventilation. Ed. Médica Panamericana, 2010.

3. Javier Muñoz Bono, Emilio Curiel Balsera, Juan Luis Galeas López. Indicaciones en ventilación mecánica no invasiva. ¿Evidencias en la bibliografía médica? Medicina Clínica (Barcelona). 2011; 136 (3):116-120. [Internet] 2014. [consultado el 28 de marzo de 2014] Disponible en: https://cspt.es/tauli/cat/Lacorporacio/elscentres/HS/webs/Urgencies/6 Info professionals/Ses sions/Sessions Bibliografiques/documents/Revision VMNI 2011.pdf

4. Bautista Villaécija O, Campaña Castillo F. Protocolo de utilización BIPAP en paciente ingresado en la unidad de Medicina Interna. NURE Investigación Núm. 65. Jul-ago 2013; 10(65):[aprox. 7 p.] [Internet] 2014. [consultado el 28 de marzo de 2014] Disponible en:

http://www.fuden.org/FICHEROS ADMINISTRADOR/PROTOCOLO/NURE65 protocolo bipap.pdf

5. M. Delgado, A. Marcos, A. Tizón, A. Carrillo. Impacto del fracaso de la ventilación no invasiva en el pronóstico de los pacientes. Subanálisis de un estudio multicéntrico. Original Research Article. Medicina Intensiva, Volume 36, Issue 9, December 2012, Pages 604-610.

6. Salvador Díaz Lobato, Sagrario Mayoralas Alises, Guillermo Montiel. Ventilación mecánica no invasiva en la agudización de las enfermedades respiratorias Review Article. Medicina Clínica, Volume 137, Issue 15, 10 December 2011, Pages 691-696.

7. Yaquelín Naranjo Vargas, Zenén Rodríguez Fernández, Yamilé Gómez Martínez. Ventilación mecánica no invasiva y acciones de enfermería en pacientes con pulmón húmedo traumático. MEDISAN vol.15 no.8 Santiago de Cuba ago. 2011. [Internet] [consultado el 28 de marzo de 2014] Disponible en: http://scielo.sld.cu/scielo.php?script=sci arttext\&pid=S1029-30192011000800010

8. M. Raurell Torredà. Impacto de los cuidados de enfermería en la incidencia de neumonía asociada a la ventilación mecánica invasiva Original Research Article Enfermería Intensiva, Volume 22, Issue 1, January-March 2011, Pages 31-38. 
9. N. Ania González, R. Goñi Viguria. Revisión de conocimientos sobre los cuidados en la prevención de la neumonía asociada a la ventilación mecánica. Enfermería Intensiva, Volume 23, Issue 4, October-December 2012, Pages 189-193.

10. Aurelio Rodríguez Fernández, Cecilia del Pozo Hessing. Ventilación mecánica no invasiva en pacientes con insuficiencia respiratoria aguda. MEDISAN vol.17 no.5 Santiago de Cuba mayo 2013. [Internet] [consultado el 28 de marzo de 2014] Disponible en:

http://scielo.sld.cu/scielo.php?pid=S1029-30192013000500003\&script=sci arttext\&tlng=pt

11. Gonzalo Segrelles Calvo, Enrique Zamora García, Rosa Girón Moreno. Ventilación mecánica no invasiva en una población anciana que ingresa en una unidad de monitorización respiratoria: causas, complicaciones y evolución al año de seguimiento Original Research Article. Archivos de Bronconeumología, Volume 48, Issue 10, October 2012, Pages 349-354.

12. G. Segrelles Calvo, E. Zamora García, R. Girón Moreno. Seguimiento al año de pacientes que ingresan en una Unidad de Monitorización Respiratoria por acidosis respiratoria que requiere ventilación mecánica no invasiva. Revista Patología Respiratoria. 2013; 16(1): 8-13. [Internet] [consultado 28 de marzo de 2014] Disponible en: http://revistadepatologiarespiratoria.org/descargas/pr_16-1 8-13.pdf

13. JM Carratalá, J Masip. Ventilación no invasiva en la insuficiencia cardiaca aguda: uso de CPAP en los servicios de urgencias. Sociedad Española de Medicina de Urgencias y Emergencias. SEMES. Emergencias 2010; 22: 49-55.

14. José Manuel Carratalá, Pere Llorens, Benjamín Brouzet et al. Ventilación no invasiva en insuficiencia cardiaca aguda perfil clínico y evolución de pacientes atendidos en un servicio de urgencias hospitalario. Emergencias: Revista de la Sociedad Española de Medicina de Urgencias y Emergencias, Vol. 22, No. 3, 2010, págs. 187-192. [Internet] [consultado el 28 de marzo de 2014] Disponible en: http://dialnet.unirioja.es/servlet/articulo?codigo $=3224210$

15. Jose Echave-Sustaeta, Lorena Comeche Casanova et al. Pronóstico tras una agudización grave de la EPOC tratada con ventilación mecánica no invasiva. Original Research Article. Archivos de Bronconeumología, Volume 46, Issue 8, August 2010, Pages 405-410.

16. Rosa Mirambeaux Villalona, Sagrario Mayoralas Alises, Salvador Díaz Lobato. Resolución de atelectasia obstructiva con ventilación mecánica no invasiva. Archivos de Bronconeumología. 2014. 